\title{
An efficient FEM-BEM procedure for the multi-frequency acoustical analysis of lined ducts containing passive components
}

\author{
R. Maréchal, E. Perrey-Debain, J.-M. Ville \& B. Nennig \\ Université Technologique de Compiègne, France
}

\begin{abstract}
This paper deals with strategies for computing efficiently the propagation of sound waves in ducts with acoustic lining at its walls. Although efficient, these treatments seem to have reached their limit and there is still a need for considering other passive techniques to reduce further the sound radiation at the duct exit. In most cases of practical interest, these added acoustics components can be modeled as acoustic cavities, which are connected to the duct and can be either purely reactive or dissipative. The assessment of the efficiency of such a system requires a precise knowledge of the acoustic field in the duct. Although standard Finite Element (FE) software could, in principle, be used for this purpose, a full FE model would be extremely demanding, particularly in the mid-frequency range and this can have a negative impact when, for instance, some efficient optimizations are needed. In the present work, we present a new numerical procedure that judiciously exploits the benefit of the FEM and the BEM approach. Firstly, a set of FE eigenmodes are computed in the cavity to produce a numerical impedance matrix connecting the pressure and the acoustic velocity on the duct wall interface. Then an integral representation for the acoustic pressure in the main duct is used. The presence of acoustic liners on the walls of the duct is taken into account via an appropriate modal decomposition of the Green's function. Typical applications involving Helmholtz resonators and Herschel-Quincke tubes are presented. We show that our algorithm allows a very fast and accurate computation of the scattering matrix of such a system with a numerical complexity that grows very mildly with the frequency.
\end{abstract}

Keywords: impedance matrix, modal decomposition, lined duct, green's function, integral equations. 


\section{Introduction}

In a large number of sound generating devices, sound waves of large amplitude are being set up inside a tube and some of the acoustic energy propagates in the duct before being radiated into the open. The practical applications of such systems range from noise transmission in vehicle exhaust systems, through ventilation and air conditioning ducts, to sound propagation in the ducted regions of turbofan aircraft engines.

The acoustic energy flow reduction techniques for the duct noise problem can be divided into two categories. Reactive techniques specifically aim to alter the duct impedance by reflecting back most of the incident acoustic wave. In most cases of practical interest, these acoustics components can be modeled as acoustic cavities, which are connected to the duct. Dissipative techniques specifically aim to absorb the sound field as it propagates down the duct. These most commonly take the form of a lining material (porous and/or perforate metal panel) placed on the walls of the duct. While these absorbent materials are known to be typically effective at relatively high frequencies, reactive techniques are best applied to low frequency noise problems. For these reasons, these techniques seem to have reached their limit when used on their own and there is still a need to consider the benefit of these two combined in order to reduce further the sound radiation at the duct exit. The assessment of the efficiency of such a system requires a precise knowledge of the acoustic field in the duct. Although standard Finite Element (FE) software could, in principle, be used for this purpose, a full FE model would be extremely demanding as the number of variables is expected to grow like $f^{d}$ ( $f$ is the frequency and $d=2,3$ the dimension of the discretized domain). To make the matter worse, the FE method is known to suffer from pollution errors, which can be avoided at the price of a very high discretization level, particularly in the medium and high frequency range [1]. This can have a negative impact when some efficient optimizations (geometry and positions of these added components for instance) are needed.

In this work, we present a new numerical procedure that judiciously exploits the benefit of the FEM and the BEM approach. The idea relies mainly on the concept of an impedance matrix that connects the pressure to the acoustic normal velocity on the duct-cavity interface. This considerably reduces the number of variables as only the interface needs to be discretized. The technique is based on the use of integral equations for the acoustic pressure with appropriate Green's functions. The theory is presented in Sections 3 and 4. The last section shows practical applications involving Helmholtz resonators [2] and Herschel-Quincke tubes [3]. The benefit of the present approach is shown both in terms of CPU time and model reduction, when compared to standard FE models.

\section{Problem statement}

The problem under consideration is illustrated in Fig. 1. It consists of a twodimensional lined main duct (domain $\Omega$ ) of height $h$ which is connected to a single 
cavity $\Omega_{c}$. The inlet and outlet pipes (regions I and II) are identical, each having rigid walls at its boundaries $\Gamma_{w}$. We wish to evaluate the scattering matrix (or Smatrix) of this acoustic system, that is given incident pressure waves $P_{\mathrm{I}}^{+}$and $P_{\mathrm{II}}^{-}$, we compute the scattered waves $P_{\mathrm{I}}^{-}$and $P_{\mathrm{II}}^{+}$. We call $\Gamma_{1}\left(\operatorname{resp} . \Gamma_{2}\right)$ the lined wall of the main duct with impedance $Z_{1}$ (resp. $Z_{2}$ ).

In the main duct, the acoustic pressure $p$ satisfies the Helmholtz equation

$$
\Delta p+k^{2} p=0 .
$$

On the lined walls, a local impedance condition is prescribed:

$$
Z_{i}=\frac{1}{Y_{i}}=\frac{p}{\mathbf{v} \cdot \mathbf{n}}, \quad i=1,2 .
$$

Note the impedance is generally function of the frequency and the acoustic normal velocity is proportional to the pressure gradient, i.e. $\partial_{n} p=i \omega \rho \mathbf{v} \cdot \mathbf{n}$ where $\mathbf{n}$ is the outward unit normal. Here, we adopt the $\mathrm{e}^{-\mathrm{i} \omega t}$-convention, $k=\omega / c$ is the wave number, $c$ the celerity, $\omega$ the angular frequency, $\rho$ the fluid density. The transmission conditions at the artificial boundaries $\Gamma_{I}$ and $\Gamma_{I I}$ are given from the pressure wave field in the inlet and outlet pipes. This is expressed as the usual modal series

$$
P_{j}^{ \pm}=\sum_{m=0}^{\infty} A_{j, m}^{ \pm} \psi_{m}^{0}(x) \mathrm{e}^{ \pm \mathrm{i} \beta_{m}^{0} z}
$$

where $j=\mathrm{I}$ or II. Here the pair $\left(\psi_{m}^{0}, \beta_{m}^{0}\right)$ defines the classical propagative (or evanescent) mode in the rigid pipe (the superscript 0 refers to the rigid wall case). Finally, we require that $p$ and its normal derivative (i.e. the normal velocity) to be continuous across the duct-cavity interface $\Gamma$.

\section{Impedance matrix: general theory}

To simplify the analysis, we will consider a cavity filled with air and acoustic losses can be taken into account via a locally reacting treatment on the cavity wall. Let

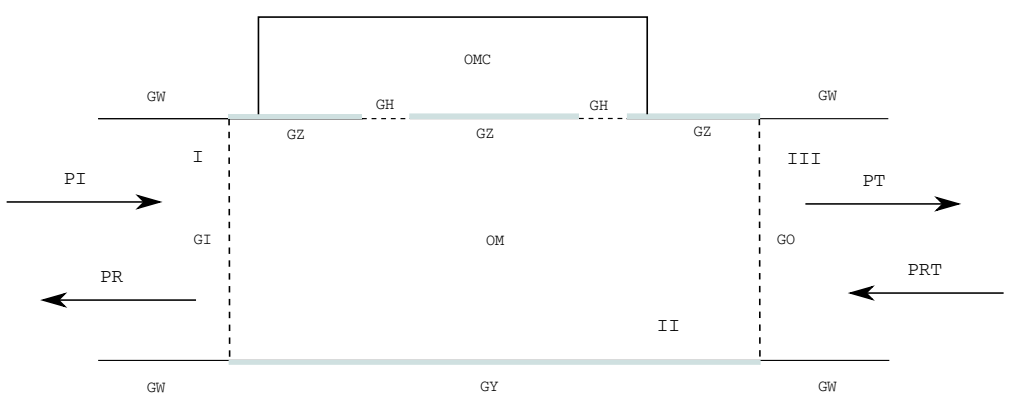

Figure 1: Main duct with a single passive component. 
us first assume that the acoustic cavity $\Omega_{c}$ is closed, that is we enforce Neumann rigid boundary conditions on the apertures $\Gamma$. Standard results show there exists a complete set of orthogonal eigenfunction $\Phi_{n}$ belonging to the spectrum of the Laplacian operator (see [4])

$$
\Delta \Phi_{n}=-k_{n}^{2} \Phi_{n}
$$

subject to the boundary conditions: $\partial_{n} \Phi_{n}=0$ on the apertures $\Gamma$ and $\partial_{n} \Phi_{n}=$ i $\rho \omega Y_{c} \Phi_{n}$ everywhere else. Here, $Y_{c}$ denotes the complex wall admittance and $\partial_{n}$ denotes differentiation along the outward normal to the cavity. An important fact to keep in mind is that the boundary conditions depends on frequency (except in the limiting case of rigid walls) so that the set of eigenfunctions $\Phi_{n}$ and eigenvalues $k_{n}$ also are functions of $\omega$. Now, any given pressure field in $\Omega_{c}$ can be written as a weighted sum of the eigenfunctions. In particular, the Green's function for the cavity, which satisfies

$$
\Delta G_{c}+k^{2} G_{c}=-\delta(\boldsymbol{x}-\boldsymbol{y})
$$

is given by the infinite series

$$
G_{c}(\boldsymbol{x}, \boldsymbol{y}, \omega)=\sum_{n=0}^{\infty} \frac{\Phi_{n}(\omega, \boldsymbol{x}) \Phi_{n}(\omega, \boldsymbol{y})}{\omega^{2}-\omega_{n}^{2}}
$$

where for the sake of clarity, we put $\omega_{n}=k_{n}(\omega) c$. Eigenfunctions are properly normalized so that application of the Green's theorem in the cavity yields the standard result

$$
p(\boldsymbol{x})=\int_{\Gamma} G_{c} \partial_{n} p \mathrm{~d} \gamma(\boldsymbol{y}) .
$$

Note that this integral formulation is not only valid for any point $\boldsymbol{x}$ inside the cavity but also on the boundary $\Gamma$. Collocating (7) on $\Gamma$ leads, after discretization, to the linear system

$$
\mathbf{p}_{\text {int }}=\mathbf{Z}(\omega) \tilde{\mathbf{F}} \partial_{n} \mathbf{p}_{\text {int }} .
$$

Here, the vector $\mathbf{p}_{\text {int }}$ (resp. $\partial_{n} \mathbf{p}_{\text {int }}$ ) simply contains the discrete nodal values of the pressure (resp. normal derivative pressure) on the duct-cavity interface and, in this context, the frequency-dependent matrix $\mathbf{Z}$ can be interpreted as an impedance matrix. The matrix $\tilde{\mathbf{F}}$ stems from discretization of the boundary integral, its exact from is not essential for the moment but we will comment on this further on. Without loss of generality, it is easy to see from (6), that this matrix admits the following truncated eigenmode decomposition

$$
\mathbf{Z}(\omega)=\left(\tilde{\boldsymbol{\Phi}} \mathbf{D}(\omega) \tilde{\boldsymbol{\Phi}}^{\mathrm{T}}+\mathbf{R}(\omega)\right)
$$

The matrix $\tilde{\boldsymbol{\Phi}}=\left(\tilde{\boldsymbol{\Phi}}_{1} \ldots \tilde{\boldsymbol{\Phi}}_{N}\right)$ contains in its columns the nodal value of the eigenfunctions $\Phi_{n}(n=0,1, \ldots, N)$ and $\mathbf{D}$ stands for the diagonal matrix with its diagonal entries: $\left(\omega_{n}^{2}-\omega^{2}\right)^{-1}$. Here the tilde symbol means that we only retain 
the nodal values of these eigenmodes on the duct-cavity interface $\Gamma$. The interest for the decomposition (9) becomes clearer when the frequency of interest is taken well below the highest modal 'resonant' frequency (i.e. $\left|\omega-\omega_{N}\right|^{-1} \ll 1$ ). In this frequency range, the correction term $\mathbf{R}$ is generally weakly dependent on the frequency, so we can take the low order Taylor expansion

$$
\mathbf{R}(\omega) \approx \mathbf{R}(\bar{\omega})+(\omega-\bar{\omega}) \frac{\partial \mathbf{R}}{\partial \omega}(\bar{\omega})+\ldots
$$

where $\bar{\omega}$ is a reference value to be givenly precisely later. Once the matrix $\mathbf{R}(\bar{\omega})$ and its derivatives have been stored, the computation of (9) becomes a very fast and simple procedure. Its efficiency relies on the numerical computation of a sufficiently large set of eigenfunctions as well as finding the residual matrix. In this work, a finite element strategy is used to discretize the exact geometry of the cavity so that no assumption is made a priori regarding the shape of the acoustic eigenmodes. We consider a finite element mesh made of linear elements (twodimensional triangles) and we denote by $\phi_{i}$ the associated piecewise linear shape function. Standard procedure leads to the following algebraic eigenmode problem:

$$
\left(\mathbf{K}+\mathbf{C}(\omega)-\omega_{n}^{2} \mathbf{M}\right) \boldsymbol{\Phi}_{n}=0
$$

where matrices involved are obtained from

$$
(\mathbf{K})_{i j}=\int_{\Omega_{c}} \nabla \phi_{i} \nabla \phi_{j} \mathrm{~d} \Omega \quad(\mathbf{M})_{i j}=\frac{1}{c^{2}} \int_{\Omega_{c}} \phi_{i} \phi_{j} \mathrm{~d} \Omega,
$$

and

$$
(\mathbf{C}(\omega))_{i j}=-\mathrm{i} \rho \omega \int_{\partial \Omega_{c} \backslash \Gamma} Y_{c}(\omega) \phi_{i} \phi_{j} \mathrm{~d} \gamma
$$

Equation (11) constitutes a generalized eigenvalue problem. Depending on the matrix size (i.e. the number of FE nodes in the whole cavity) and the number of modes retained in the series, the evaluation of the impedance matrix might be computationally expensive as this needs to be done for each frequency. This point will be discussed at the end of the section.

At this stage, we shall simplify the analysis a bit further by considering rigid boundary conditions only (i.e. we put $Y_{c}=0$ ). In this case, the problem becomes linear in $\lambda=\omega^{2}$, i.e.

$$
\mathbf{A}_{0}\left(\lambda_{n}^{0}\right) \boldsymbol{\Phi}_{n}^{0}=0 \quad \text { where } \quad \mathbf{A}_{0}(\lambda)=\mathbf{K}-\lambda \mathbf{M}
$$

Here the superscript 0 refers to the rigid wall case. A first set of mode $(n=$ $0,1, \ldots, N)$ is then computed using appropriate large sparse eigenvalue problem solvers where it is understood that the truncation order $N$ is taken well below the 
FE matrix size. Now, finding the residual matrix is a bit more tricky as we first need to go back to original direct problem:

$$
\mathbf{A}_{0}(\lambda) \mathbf{p}=\mathbf{F} \partial_{n} \mathbf{p}_{\text {int }},
$$

where the vector $\mathbf{p}$ contains the value of the pressure at all nodes of the FE mesh. To ease the demonstration, we proceed to an appropriate elements reordering so that the rectangular matrix $\mathbf{F}$ looks like

$$
\mathbf{F}=\left(\begin{array}{c}
\tilde{\mathbf{F}} \\
\mathbf{0}
\end{array}\right)=\mathbf{I}_{\Gamma} \tilde{\mathbf{F}} \quad \text { with } \quad(\tilde{\mathbf{F}})_{i j}=\int_{\Gamma} \phi_{i} \phi_{j} \mathrm{~d} \gamma .
$$

Here $\mathbf{I}_{\Gamma}$ denotes the identity matrix for nodes on the interface only. Inverting (15) shows that the impedance matrix has the alternative form

$$
\mathbf{Z}_{0}(\lambda)=\mathbf{I}_{\Gamma}^{\mathrm{T}} \mathbf{A}_{0}^{-1}(\lambda) \mathbf{I}_{\Gamma}
$$

The residual matrix is then computed simply via

$$
\mathbf{R}_{0}=\mathbf{Z}_{0}-\tilde{\boldsymbol{\Phi}}^{0} \mathbf{D}\left(\tilde{\boldsymbol{\Phi}}^{0}\right)^{\mathrm{T}}
$$

and the first order derivative can be computed from

$$
\frac{\partial \mathbf{R}_{0}}{\partial \lambda}=\mathbf{I}_{\Gamma}^{\mathrm{T}} \mathbf{A}_{0}^{-1} \mathbf{M} \mathbf{A}_{0}^{-1} \mathbf{I}_{\Gamma}-\tilde{\boldsymbol{\Phi}}^{0} \frac{\partial \mathbf{D}}{\partial \lambda}\left(\tilde{\boldsymbol{\Phi}}^{0}\right)^{\mathrm{T}} .
$$

Note that (i) the $\omega$-derivative is recovered from $\partial_{\lambda} \mathbf{R}_{0}=2 \omega \partial_{\omega} \mathbf{R}_{0}$ and (ii) the $\lambda$ derivative of the diagonal matrix $\mathbf{D}$ is an easy task as eigenvalues $\lambda_{n}^{0}=\left(\omega_{n}^{0}\right)^{2}$ are frequency-independent in the rigid wall case. Now exploiting the symmetry of $\mathbf{A}_{0}$, we see that $\mathbf{I}_{\Gamma}^{\mathrm{T}} \mathbf{A}_{0}^{-1}=\left(\mathbf{A}_{0}^{-1} \mathbf{I}_{\Gamma}\right)^{\mathrm{T}}$. Thus the important point to make is that the full inversion of $\mathbf{A}_{0}$ is not needed here. Only the first columns of $\mathbf{A}_{0}^{-1}$ corresponding to the interface nodes are active and these can be efficiently computed by solving successively $\mathbf{A}_{0} \mathbf{v}_{i}=\mathbf{e}_{i}$ with appropriate sparse solvers ( $\mathbf{e}_{i}$ is the column vector with zero elements with the unity on the $i$ th line). It is clear that the reference value $\bar{\lambda}$ must be chosen away from the resonant value $\lambda_{n}^{0}$ to guarantee that the inversion of $\mathbf{A}_{0}$ is not spoiled by round off error. In this work we take $\bar{\lambda}=\lambda_{1}^{0} / 2$ (the value $\bar{\lambda}=\lambda_{0}^{0}=0$ is proscribed here as it corresponds to the rigid motion resonance).

Now going back to the more general frequency dependent problem (11), the large size matrix system can be considerably reduced by searching the lossy eigenmode in the basis of the rigid modes, i.e.

$$
\boldsymbol{\Phi}_{n}=\boldsymbol{\Phi}^{0} \mathbf{q}_{n}
$$

Now by construction we have that

$$
\left(\boldsymbol{\Phi}^{0}\right)^{\mathrm{T}} \mathbf{M} \boldsymbol{\Phi}^{0}=\mathbf{I} \quad \text { and } \quad\left(\boldsymbol{\Phi}^{0}\right)^{\mathrm{T}} \mathbf{K} \boldsymbol{\Phi}^{0}=\boldsymbol{\Lambda}^{0},
$$


where $\Lambda^{0}$ is a diagonal matrix containing the rigid wall eigenvalues $\lambda_{m}^{0} m=$ $0,1, \ldots, N$ in ascending order. The eigenvalue problem becomes

$$
\left(\boldsymbol{\Lambda}^{0}+\left(\boldsymbol{\Phi}^{0}\right)^{\mathrm{T}} \mathbf{C}(\omega) \boldsymbol{\Phi}^{0}-\omega_{n}^{2}\right) \mathbf{q}_{n}=0 .
$$

Note that in most cases, high order modes are expected to behave almost like rigid wall modes (i.e. $\omega_{n}^{2} \rightarrow\left(\omega_{n}^{0}\right)^{2}$ and $\boldsymbol{\Phi}_{n} \rightarrow \boldsymbol{\Phi}_{n}^{0}$ as $n$ increases) and the matrix $\mathbf{C}(\omega)$ can be treated as a perturbation term [4], this fact will be illustrated in the next section. Finally, the residual matrix is obtained following the same procedure from

$$
\mathbf{Z}(\bar{\omega})=\mathbf{I}_{\Gamma}^{\mathrm{T}} \mathbf{A}^{-1}(\bar{\omega}) \mathbf{I}_{\Gamma} \quad \text { with } \quad \mathbf{A}(\omega)=\left(\mathbf{K}+\mathbf{C}(\omega)-\omega^{2} \mathbf{M}\right) .
$$

The first order derivative matrix is more difficult to obtain as all quantities involved are now frequency dependent and this will not be dealt with here.

\section{S-matrix of the acoustic system}

In the main duct, the theory starts by introducing the lined-walled duct Green's function satisfying the usual modal radiation condition on both ends of the main duct, i.e.

$$
G\left(\boldsymbol{x}, \boldsymbol{x}_{0}\right)=\sum_{n=0}^{\infty} \frac{\psi_{n}(x) \psi_{n}\left(x_{0}\right)}{-2 \mathrm{i} \beta_{n}} \mathrm{e}^{\mathrm{i} \beta_{n}\left|z-z_{0}\right|}
$$

where $\boldsymbol{x}=(x, z)$ and $\boldsymbol{x}_{0}=\left(x_{0}, z_{0}\right)$ are two points in the propagative domain $\Omega$. Function $\psi_{n}$ is the transverse mode, i.e.

$$
\psi_{n}^{\prime \prime}+\alpha_{n}^{2} \psi_{n}=0
$$

and satisfying the lined-wall conditions (2) on both sides $x=0$ and $x=h$ (here the prime signifies derivation with respect to the transverse coordinate $x$ ). Again, such an eigenmode problem could be solved using FE techniques. However, an analytical approach is adopted here. It suffices to look for solutions of the form $\psi=A \cos (\alpha x)+B \sin (\alpha x)$. The transverse 'resonant' wavenumbers are found so that the $2 \times 2$ system

$$
\mathbf{M}(\alpha)\left(\begin{array}{c}
A \\
\alpha B
\end{array}\right)=0
$$

admits non trivial solutions. It is more convenient for the analysis to search the axial wavenumber $\beta$, so we put $\alpha=\sqrt{k^{2}-\beta^{2}}$ and solve the dispersion equation

$$
g(\beta)=\operatorname{det} \mathrm{M}\left(\sqrt{k^{2}-\beta^{2}}\right)=0 .
$$

Using the fact that $g$ is analytic over the complex plane (in $\beta$ ), low order modes are numerically calculated with a technique described by Kravanja and Van Barel [5] and already used by the authors for the modeling of dissipative silencers [6]. 
Higher order modes are recovered using asymptotic expansions. This is easily achieved by expanding the transverse wavenumbers as the truncated series

$$
\alpha_{n} h=\alpha_{n}^{0} h+\sum_{k=1}^{K} \frac{a_{k}}{n^{k}},
$$

where $\alpha_{n}^{0}=n \pi / h$ are the transverse wavenumbers of the rigid wall duct. This simple example reveals that lined wall modes behave almost like the rigid ones when $n$ is taken sufficiently large. In our applications, we observed that $n>10$ was a sufficient condition for the series (28) to converge quickly with a relatively small number of terms $(K=5)$.

Using the Green's theorem, the pressure anywhere in the lined duct ( $\Omega$ domain) is given via the integral representation

$$
p=\int_{\Gamma_{\mathrm{I}} \cup \Gamma_{\mathrm{II}}}\left(G \partial_{n} p-p \partial_{n} G\right) \mathrm{d} \gamma+\int_{\Gamma} G\left(\partial_{n} p-\mathrm{i} \rho \omega Y_{2} p\right) \mathrm{d} \gamma
$$

The discretization of this equation is carried out in two steps. First, collocating (29) at the FEM nodes of the duct-cavity interface leads to

$$
\mathbf{K}_{\Gamma, \Gamma} \partial_{n} \mathbf{p}_{\text {int }}+\mathbf{K}_{\Gamma, \mathrm{I}} \mathbf{A}_{\mathrm{I}}^{-}+\mathbf{K}_{\Gamma, \mathrm{II}} \mathbf{A}_{\mathrm{II}}^{+}=\mathbf{F}_{\Gamma, \mathrm{I}} \mathbf{A}_{\mathrm{I}}^{+}+\mathbf{F}_{\Gamma, \mathrm{II}} \mathbf{A}_{\mathrm{II}}^{-}
$$

where vectors $\mathbf{A}_{j}^{ \pm}$contain the modes amplitudes $A_{j, m}^{ \pm},(j=\mathrm{I}, \mathrm{II})$. The first block matrix

$$
\mathbf{K}_{\Gamma, \Gamma}=-\mathbf{Z}(\omega) \tilde{\mathbf{F}}-\mathbf{G}-\mathrm{i} \rho \omega Y_{2} \mathbf{G} \mathbf{Z}(\omega) \tilde{\mathbf{F}}
$$

stems from the self interaction of the acoustic pressure at the interface. Here the Green matrix $\mathbf{G}$ stems from the discretization of the second integral in (29). Other matrices are built by simply substituting $p=P_{j}^{+}+P_{j}^{-}$and $\partial_{n} p=\partial_{n}\left(P_{j}^{+}+P_{j}^{-}\right)$ with $(j=\mathrm{I}, \mathrm{II})$ in the first integral of (29). This operation requires the computation of the coupling coefficients $C_{m n}$ given by the overlap integrals

$$
C_{m n}=\int_{0}^{h} \psi_{m}^{0}(x) \psi_{n}(x) \mathrm{d} x,
$$

which also arise in standard mode matching techniques [6]. The system (29) is completed by taking the evaluation point in the integral equation on the inlet and outlet boundaries. An additional set of equations is then produced by projecting (29) onto the hard-wall modes basis to give

$$
\mathbf{K}_{j, \Gamma} \partial_{n} \mathbf{p}_{\text {int }}+\mathbf{K}_{j, \mathrm{I}} \mathbf{A}_{\mathrm{I}}^{-}+\mathbf{K}_{j, \mathrm{II}} \mathbf{A}_{\mathrm{II}}^{+}=\mathbf{F}_{j, \mathrm{I}} \mathbf{A}_{\mathrm{I}}^{+}+\mathbf{F}_{j, \mathrm{II}} \mathbf{A}_{\mathrm{II}}^{-}
$$

for both boundaries $j=\mathrm{I}$ and II. Finally, the scattered modes amplitudes $\mathbf{A}_{\mathrm{I}}^{-}$and $\mathbf{A}_{\text {II }}^{+}$are found by solving (29) together with (32). In practice, the summation in (3) is limited to the number of propagative modes as well as some evanescent modes which are included to ensure a precise approximation of the pressure field in the inlet and outlet boundaries. Thus, the scattering matrix system is of a relatively small size in the present study. 


\section{Results and concluding remarks}

The first example concerns the effect of a HQ tube placed in the lined section of the duct (see Fig. 2). The width of the main duct is $h=2 a=0.04859 \mathrm{~m}$ and the length of the liner is $0.6 \mathrm{~m}$. The results are presented with respect to the dimensionless variable $k a$. The study is carried out from very low frequency up to $k a=2.23$ (which corresponds to $5000 \mathrm{~Hz}$ ) with a stepsize of $1 \mathrm{~Hz}$. In the overall frequency range, the incident pressure is a plane wave. The first cut-off frequency occurs at $k a=\pi / 2$. For the lined wall, the impedance value are chosen as to be in line with perforate plates encountered in the aeronautic industry. Thus, we take $Z_{1}=\rho c(2+2 \mathrm{i})$ and $Z_{2}=\rho c(1+1 \mathrm{i})$. Note that the expected frequency dependence of the impedance is not taken into account here but this can be easily included in the analysis.

Here, we are interested in the Transmission Loss (TL) is defined as the ratio of transmitted acoustic power with respect to the incident one, that is

$$
\mathrm{TL}=-10 \log _{10}\left(\frac{1}{\beta_{0}^{0}\left|A_{\mathrm{I}, 0}^{+}\right|^{2}} \sum_{m \geq 0} \beta_{m}^{0}\left|A_{\mathrm{II}, m}^{+}\right|^{2}\right),
$$

where the summation is limited to propagative modes only. In order to validate the method, a full finite element model is used. Radiation conditions at both ends of the duct have been implemented using the DtN map [7]. In Fig. 3 are plotted the TL calculated from our method and the FE model. The HQ tube is about $27 \mathrm{~cm}$ long with a width of $4.6 \mathrm{~cm}$ and the first 250 modes are included for the impedance matrix calculation (the FE mesh contains about 2,500 elements). The very good agreement validates the present method and the small discrepancies noticeable at high frequency are thought to be due to the FE model which starts loosing accuracy. The number of FE nodes is very large compared to number of variables used in our model (i.e. the number of nodes at the interface + the number of modes in the summation in (3)) which does not exceed 30, this is reported in Table 1. At higher frequency, this number is expected to grow very mildly with the frequency whereas the FE model would quickly become intractable because of the

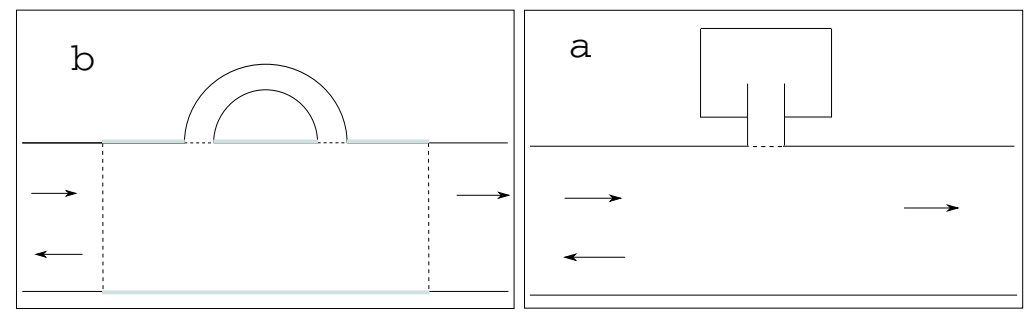

Figure 2: Typical acoustic components. (a): HQ tube connected to a lined section of a duct (gray color). (b): Helmholtz resonator connected to a rigid-wall duct. 


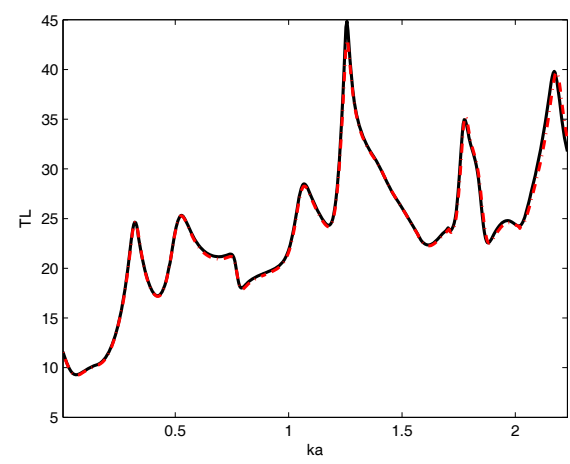

Figure 3: HQ tube in a lined duct : —— our method, - - - full FE model.

Table 1: Comparison of the proposed method with a full FE model. (*) time corresponding to 5,000 TL calculation on a single PC using MATLAB.

\begin{tabular}{cccc}
\hline \hline & CPU time $^{(*)}$ & FE mesh & Total dof \\
\hline HQ tube & $10 \mathrm{~min}$ & 2,500 & 30 \\
Helm. resonator & $30 \mathrm{~s}$ & 5,000 & 10 \\
full FE model & $75 \mathrm{~min}$ & 12,000 & 12,000 \\
\hline \hline
\end{tabular}

computational overhead. Note that (i) a large part of the CPU time is due to the calculation of the roots of the dispersion equation (27) and this could be largely reduced by using fastest methods such as the Newton-Raphson method and (ii) in all calculations, the modal series for the lined duct Green's function is truncated by keeping the first 250 terms in the series to ensure that the diagonal dominant coefficients of the interface-interface interaction matrix $\mathbf{K}_{\Gamma, \Gamma}$ are computed with sufficient accuracy.

The second example concerns the effect of one Helmholtz resonator connected to a rigid wall duct. This example is taken from [2] and shown in Fig. 2. For the sake of illustration, Fig. 4 shows typical eigenmode patterns for three resonators with different neck extensions (in order of appearance: straight, with conical contraction and with conical expansion). Again, a comparison with a full FE model gives perfect agreements as shown in Fig. 5. Here, the main duct is rigid and there is no need to find the lined duct Green's function, this explains the very low CPU time (30 s only). The right figure shows the effect of the extended necks on the TL.

Through these examples, the method presented in this work has shown to be extremely beneficial, both in term of CPU time and model reduction, when compared to standard FE models. Work is on going by the present authors to extend 

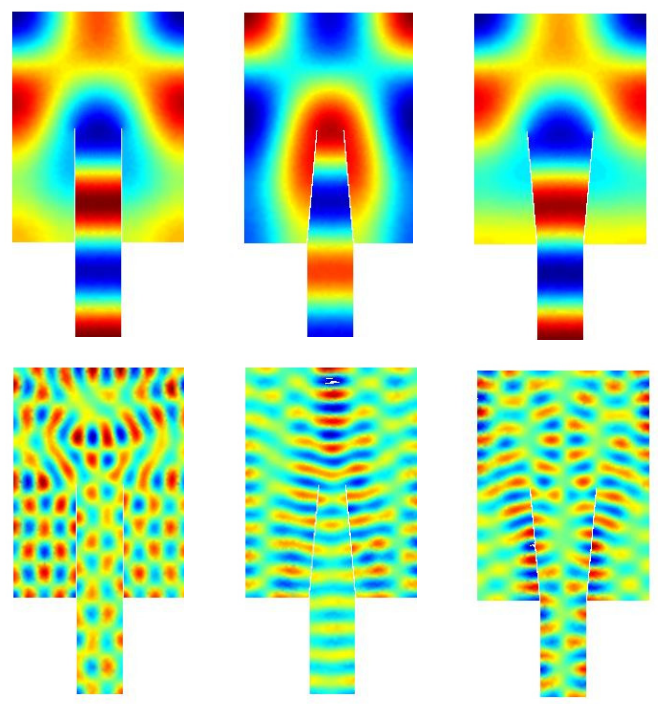

Figure 4: Eigenmodes in the Helmholtz resonators, top: Mode \#13 (around 2,800 $\mathrm{Hz}$ ) and bottom: Mode \#215 (around 15,400 Hz).
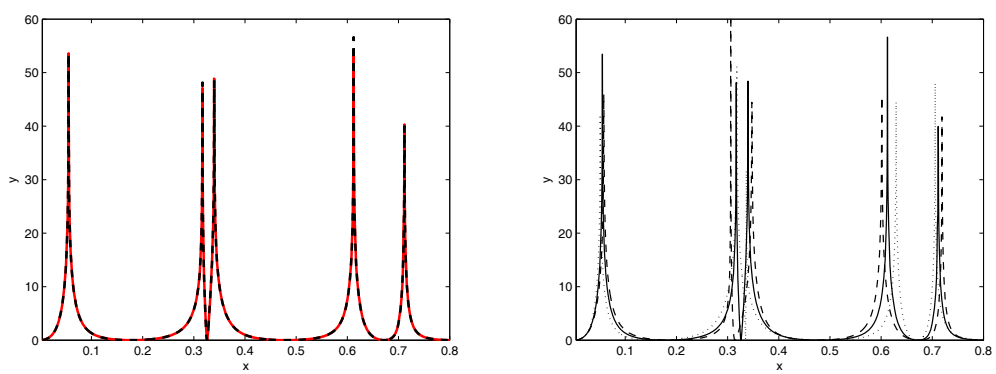

Figure 5: Left: Helmholtz resonator with straight extended neck connected to a rigid wall duct : - our method, - - - full FE model. Right: Influence of the extended necks on the Transmission Loss; solid line: straight, dashed line: with conical expansion, dotted line: with conical contraction.

the method for more realistic 3D configurations. The presence of an absorbent material in the cavity will also be considered for future work. Finally, we think that the method proposed in this work could be extended for the analysis of other noise reduction techniques such as dissipative silencers for instance. 
110 Boundary Elements and Other Mesh Reduction Methods XXXII

\section{References}

[1] Gerdes, K. \& Ihlenburg, F., On the pollution effect in FE solutions of the 3DHelmholtz equation. Comput Methods Appl Mech Engrg, 170(1), pp. 155 $172,1999$.

[2] Selamet, A. \& Lee, I., Helmholtz resonator with extended neck. J Acoust Soc Am, 113(4), pp. 1975-1985, 2003.

[3] Selamet, A. \& Dickey, N., The Herschel-Quincke tube: A theoretical, computational, and experimental investigation. J Acoust Soc Am, 96(5), pp. $3177-$ 3185, 1994.

[4] Morse, P.M. \& Ingard, K.U., Theoretical Acoustics. Princeton University Press, 1968.

[5] Kravanja, P. \& Van Barel, M., Computing the Zeros of Analytic Functions. Springer, 2000.

[6] Nennig, B., Perrey-Debain, E. \& Ben Tahar, M., Mode matching method for cylindrical dissipative silencers with poroelastic material. The 9th Int Conf on Mathematical and Numerical Aspects of Waves, 2009.

[7] Harari, I., Patlashenko, I. \& Givoli, D., Dirichlet-to-neumann maps for unbounded wave guides. J Comput Phys, 143(1), pp. 200-223, 1998. 